\title{
Brief Notes on The Possibility of Copying Qubits in Quantum Systems
}

\author{
ARTYOM GRIGORYAN ${ }^{1}$, SOS AGAIAN ${ }^{2}$ \\ ${ }^{1}$ Electrical and Engineering Department, \\ The University of Texas at San Antonio, U.S.A. \\ ${ }^{2}$ Computer Science Department, \\ City University of New York / CSI, U.S.A.
}

\begin{abstract}
Copying the quantum states is contradictory to classical information processing since the fundamental difference between classical and quantum information is that while classical information can be copied perfectly, quantum information cannot. However, this statement does not rule out the risk of building a device that can reproduce a set of quantum states. This paper investigates the naturally arising question of how well or under what conditions one can copy and measure an arbitrary quantum superposition of states. The CNOT and XOR operation-based quantum circuit is presented that exhibits entanglement of states and allows for measuring the doubled qubits.
\end{abstract}

Key-Words: - Quantum computing, Quantum bits, Entanglement state, Qubit duplication.

Received: April 21, 2021. Revised: January 8, 2022. Accepted: January 26, 2022. Published: February 10, 2022.

\section{Introduction}

As In 1982, Wootters and Zurek showed that no unitary process could create exact copies of arbitrary quantum states [1]. The statement that nonorthogonal quantum states cannot be copied was also made by Dieks [2], and new no-cloning theorems can be found in [3,4]. Many publications exist devoted to the no-cloning theorem that uses the linearity and unitarity of the copying transformations [5-7], perfect cloning of no commuting mixed states [8], perfect cloning with assistance [9], multiple copying of qubits $[10,11]$, supplementary information [12], the additional measurement for probabilistic quantum cloning $[13,14]$, cloning of identical mixed qubits [15], cloning with the local operation and classical communication [16], and unclonable encryption [17]. With such an assertion, quantum computing theory has become particularly distinctive from computing on traditional computers. In addition, such theorems were used to justify the security of quantum cryptography $[18,19]$.

The main goal of this work is to show that copies of qubits can be nested in other more complex states of systems with a large number of qubits, from where they can be measured and processed. We illustrated it, by presenting a quantum circuit that exhibits entanglement of states and allows for measuring the doubled qubits.

The rest of the paper is organized in the following way. Section 2 discusses the well-known statement about the impossibility of copying qubits. Section 3 analyses a simple quantum circuit with two CNOT and XOR operations for nesting the doubled qubits in 3- qubit. Such a circuit allows us to observe and measure the duplicated states of a qubit.

\section{Problem Formulation}

In this section, we discuss the known statement about copying qubits. The unitary operator $U$ for performing such a copy of an individual qubit in a superposition

$$
|\varphi\rangle=a|0\rangle+b|1\rangle=\left[\begin{array}{l}
a \\
b
\end{array}\right]
$$

is considered to be the 2-qubit operator

$$
\begin{gathered}
U(|\varphi\rangle|0\rangle) \triangleq U(|\varphi\rangle \otimes|0\rangle)=|\varphi\rangle^{2}=|\varphi\rangle|\varphi\rangle \\
\triangleq|\varphi\rangle \otimes|\varphi\rangle .
\end{gathered}
$$

Here, the amplitudes are such that $a^{2}+b^{2}=1$ (or $|a|^{2}+|b|^{2}=1$ in the complex case). Also, $|0\rangle$ and |1) denote the quantum computational basis states of the single qubit,

$$
|0\rangle=\left[\begin{array}{l}
1 \\
0
\end{array}\right], \quad|1\rangle=\left[\begin{array}{l}
0 \\
1
\end{array}\right]
$$

and $\otimes$ is the operation of the tensor product, or Kronecker product, of vectors.

When measuring the qubit, by using the Hermitian projection on the basis $|0\rangle$ and $|1\rangle$, the probability of the outcome $|0\rangle$ is $a^{2}$ and the probability of outcome $|1\rangle$ is $b^{2}$. The axioms of quantum mechanics 
demonstrate that after the measurement, a qubit is collapsed to the measured basis state so that a qubit will be destroyed in this measurement. Thus, we may perhaps state the well-known no-cloning theorem: no quantum procedure exists that can reproduce perfectly an arbitrary quantum state. Therefore, the measurement is irreversible; in contrast, it is pretty easy to copy information, even in a reversible manner in classical computers. It means that using the wellknown teleportation protocol, we may create a perfect replica of the original qubit, but this will be at the cost of destroying information encoded in the original qubit $[10,18]$. Thus, a) if we are only interested in producing imperfect copies, then it is possible to design machines (actually, to find unitary transformations) that can copy quantum states, b) if we do two identical copies, then the quality of these copies depends on the input state; and c) we may formulate the quantum copying problem goal is to produce a copy of the initial qubit, which is as close as possible to the original state, while the output state of the original qubit is minimally disturbed [18]. However, this theorem does not rule out the possibility of building a device that can copy a particular set of orthonormal quantum states [20]. In quantum computing, the CNOT operation does not allow copying the qubits, as in traditional computing, when copying the bits. In a digital computer, when copying a bit, a new cell is allocated in the computer's memory, the value of the bit is read, and then this value is written to the cell. Such a read-write-out procedure is likely to take place in quantum systems. Undoubtedly, other operators and quantum circuits are needed here. For each state of a qubit, probably somewhere in space, an identical state is reproduced. Maybe it is not in its pure form, but in some entangled state with other qubits. In other words, it is possible that such copies can be nested in other shells of systems with a large number of qubits, from where they can be measured and processed.

\subsection{No Cloning Qubits}

Let us consider the common calculations in the statement of copying qubits. We consider the qubit in the Hadamard superposition

$$
|\varphi\rangle=(|0\rangle-|1\rangle) / \sqrt{2} .
$$

If a unitary transform $U$ copies this qubit and it is a linear operator, we obtain two qubits in the following state:

$$
\begin{array}{r}
U(|\varphi\rangle|0\rangle)=\frac{1}{\sqrt{2}}(U(|0\rangle|0\rangle)-U(|1\rangle|0\rangle)) \\
=\frac{1}{\sqrt{2}}(|0\rangle|0\rangle-|1\rangle|1\rangle) \\
=\frac{1}{\sqrt{2}}(|0,0\rangle-|1,1\rangle) .
\end{array}
$$

The state of doubled qubits is

$$
\begin{aligned}
|\varphi\rangle^{2}=|\varphi\rangle|\varphi\rangle & =\left(\frac{|0\rangle-|1\rangle}{\sqrt{2}}\right) \otimes\left(\frac{|0\rangle-|1\rangle}{\sqrt{2}}\right) \\
& =\frac{1}{2}(|0,0\rangle+|1,1\rangle-|0,1\rangle \\
& -|1,0\rangle) .
\end{aligned}
$$

Thus, we obtain different 2-qubit quantum superpositions $U(|\varphi\rangle|0\rangle$ and $|\varphi\rangle|\varphi\rangle$. What we use in the above calculations is the assumption that if an unitary operator copies a single qubit in the superposition $|\varphi\rangle$, then it copies any other superposition $|\psi\rangle=c|0\rangle+d|1\rangle$ of the qubit, i.e.,

$\underbrace{U(|\varphi\rangle|0\rangle)=|\varphi\rangle|\varphi\rangle} \rightarrow \underbrace{U(|\psi\rangle|0\rangle)=|\psi\rangle|\psi\rangle}$.

\subsubsection{Matrix of the Transformation}

We can consider the quantum states $|\varphi\rangle|0\rangle$ and $|\varphi\rangle|\varphi\rangle$ as 4-D vectors,

$$
|\varphi\rangle|0\rangle=|\varphi, 0\rangle=a|00\rangle+b|10\rangle=\left[\begin{array}{l}
a \\
0 \\
b \\
0
\end{array}\right]
$$

and

$$
\begin{gathered}
|\varphi\rangle|\varphi\rangle=|\varphi, \varphi\rangle=a^{2}|00\rangle+a b(|01\rangle+|10\rangle) \\
+b^{2}|11\rangle=\left[\begin{array}{l}
a^{2} \\
a b \\
a b \\
b^{2}
\end{array}\right] .
\end{gathered}
$$

We are looking for a $4 \times 4$ unitary matrix $\boldsymbol{U}$, such that

$$
\boldsymbol{U}\left[\begin{array}{l}
a \\
0 \\
b \\
0
\end{array}\right]=\left[\begin{array}{llll}
t_{0,0} & t_{0,1} & t_{0,2} & t_{0,3} \\
t_{1,0} & t_{1,1} & t_{1,2} & t_{1,3} \\
t_{2,0} & t_{2,1} & t_{2,2} & t_{2,3} \\
t_{3,0} & t_{3,1} & t_{3,2} & t_{3,3}
\end{array}\right]\left[\begin{array}{l}
a \\
0 \\
b \\
0
\end{array}\right]=\left[\begin{array}{l}
a^{2} \\
a b \\
a b \\
b^{2}
\end{array}\right]
$$

It is clear that the coefficients of such a matrix will be defined by the values of inputs, $a$ and $b$. Unitary transformations for copying qubits are parameterized by amplitudes of quantum superpositions of states of the qubits. They are not universal, i.e., they cannot copy any qubits. It is clear that additional operators are needed to complete this task. The only operators that can be used in additional to the unitary transforms are operators of measurement, or 
projection operators. However, in order to use them, we need to first include the required 2-qubit in a system with a large number of qubits.

\section{Quantum Circuit for Nesting Doubled Qubits}

This section presents a method of nesting the doubled qubits in a larger state. In other words, we discuss the circuit which might be used to calculate the doubled qubits in arms of 3-qubit. The measurement and separation of states of doubled qubits are described.

Let us consider a qubit in the state $|\varphi\rangle=a|0\rangle+$ $b|1\rangle$ with the required condition that $|\alpha|^{2}+|b|^{2}=$ 1. These coefficients are considered real. The duplicated copy of this state is the 2-qubit state

$$
\left|\varphi^{2}\right\rangle \triangleq|\varphi\rangle|\varphi\rangle=a^{2}|00\rangle+b^{2}|11\rangle+a b|01\rangle+
$$
$a b|10\rangle$.

When applying the CNOT operator (X) with control qubit $|\varphi\rangle$ and controlling (target) state $|0\rangle$, the result is the 2-qubit state

$$
\begin{aligned}
|\psi\rangle \triangleq X[|\psi\rangle,|0\rangle]= & X[a|0\rangle+b|1\rangle,|0\rangle]= \\
& a|00\rangle+b|11\rangle,
\end{aligned}
$$

as it is illustrated in Fig. 1. This operation changes the $2^{\text {nd }}$ qubit state $|0\rangle$ to $|1\rangle$, when the control qubit is $|1\rangle$, i.e., $X[|1\rangle,|0\rangle]=|1\rangle|1\rangle=|11\rangle$. Except for the cases when $a=0$ and $b=0$, the states $\left|\varphi^{2}\right\rangle$ and $|\psi\rangle$ are different. Thus, the qubit in its general state is not copying by this circuit with the CNOT gate.

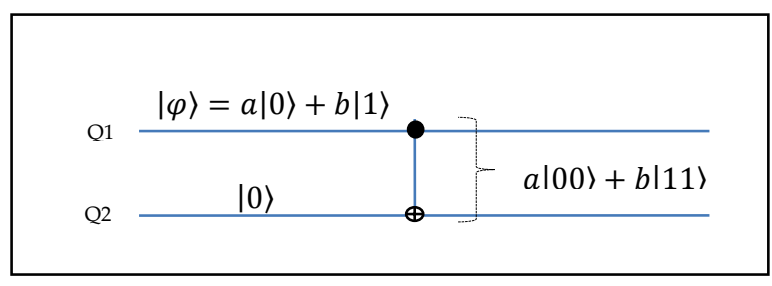

Figure 1 The circuit with the CNOT operation.

Now, we apply the second CNOT operator with a new control qubit in the state $|\phi\rangle=(|0\rangle+$ $|1\rangle) / \sqrt{2}$. This qubit can be obtained, by using the Walsh-Hadamard gate $H$ on the basis state $|0\rangle$,

$$
H|0\rangle=\frac{1}{\sqrt{2}}\left[\begin{array}{rr}
1 & 1 \\
1 & -1
\end{array}\right]|0\rangle=\frac{|0\rangle+|1\rangle}{\sqrt{2}} .
$$

The target is the second qubit of the 2-qubit state $|\psi\rangle=a|00\rangle+b|11\rangle$. The result of this operation is the following superposition of 3-qubit (without coefficient $1 / \sqrt{2}$ ):

$$
\begin{aligned}
|\chi\rangle=X[|\phi\rangle,|\psi\rangle]_{2} & \\
& =X[|0\rangle+|1\rangle, a|00\rangle+b|11\rangle]_{2} \\
& =X[|0\rangle, a|00\rangle+b|11\rangle]_{2} \\
& +X[|1\rangle, a|00\rangle+b|11\rangle]_{2} \\
=a|000\rangle+ & b|011\rangle+a|101\rangle+b|110\rangle .
\end{aligned}
$$

We consider the 3 -qubit permutation $(1,5)(2,6)$, for which we will use the gate shown in Fig. 2 in part (a) and call it the 2-XOR operator. This permutation is

$$
P_{(1,5),(2,6)}:(0,1,2,3,4,5,6,7) \rightarrow(0,5,6,3,4,1,2,7) .
$$

The matrix of this permutation can be written as

$$
\begin{gathered}
\boldsymbol{P}=\left[\begin{array}{ll}
\boldsymbol{A} & \boldsymbol{B} \\
\boldsymbol{B} & \boldsymbol{A}
\end{array}\right], \quad \boldsymbol{A}=\left[\begin{array}{llll}
1 & 0 & 0 & 0 \\
0 & 0 & 0 & 0 \\
0 & 0 & 0 & 0 \\
0 & 0 & 0 & 1
\end{array}\right], \\
\boldsymbol{B}=\left[\begin{array}{llll}
0 & 0 & 0 & 0 \\
0 & 1 & 0 & 0 \\
0 & 0 & 1 & 0 \\
0 & 0 & 0 & 0
\end{array}\right] .
\end{gathered}
$$

The logic element in this figure is not a Toffoli gate over 3-qubit state, which performs the permutation $(6,7)$. The circuit representation of the Toffoli gate is shown in part (b).

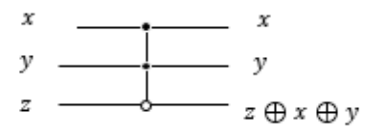

(a)

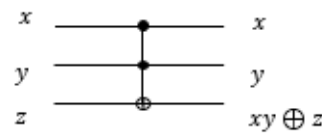

(b)
Figure 2 Circuit representation of the 3-qubit permutations (a) $(1,5)(2,6)$ and (b) $(6,7)$.

Applying this operator on 3-qubit in superposition of Eq. 8, we obtain the following state:

$$
\begin{aligned}
|\xi\rangle=\underbrace{a|000\rangle+b|010\rangle}+\underbrace{a|100\rangle+b|110\rangle} & \\
& +\underbrace{a|00\rangle+b|01\rangle}|0\rangle
\end{aligned}
$$

It is a superposition of the first four basis states $|0\rangle$, $|1\rangle,|2\rangle$, and $|3\rangle$. Now, considering the normalization coefficient $1 / \sqrt{2}$, we obtain 


$$
|\xi\rangle=\frac{a|00\rangle+b|01\rangle}{\sqrt{2}}|0\rangle+\frac{a|1\rangle+b|11\rangle}{\sqrt{2}}|0\rangle,
$$

One can note that the second qubit is in state $|\varphi\rangle=$ $a|0\rangle+b|1\rangle$. The abstract circuit for calculating this 3 -qubit $|\xi\rangle$ is given in Fig. 3 (in Appendix). The same diagram in compact form is shown in Fig. 4 (see Appendix).

Now, we consider the doubled qubits $|\varphi\rangle^{2}$. When measuring the first qubit of $|\varphi\rangle^{2}$, we obtain the state $\left|\left(\varphi^{2}\right)_{0}\right\rangle=a|00\rangle+b|01\rangle$, with the probability $a^{2}$, and the state $\left|\left(\varphi^{2}\right)_{1}\right\rangle=a|10\rangle+b|11\rangle$ with probability $b^{2}$. As follows from Eq. 9, the 3-qubit superposition $|\xi\rangle$ can be written as

$$
\begin{aligned}
|\xi\rangle=\frac{\left|\left(\varphi^{2}\right)_{0}\right\rangle|0\rangle+\left|\left(\varphi^{2}\right)_{1}\right\rangle|0\rangle}{\sqrt{2}} \\
=\frac{\left|\left(\varphi^{2}\right)_{0}\right\rangle+\left|\left(\varphi^{2}\right)_{1}\right\rangle}{\sqrt{2}}|0\rangle .
\end{aligned}
$$

Measuring the first qubit of $|\xi\rangle$, we obtain with the same probability 0.5 the following two states. If the measured qubit state is 0 , the state will be

$$
\begin{gathered}
|\xi\rangle_{0}=a|000\rangle+b|010\rangle=(a|00\rangle+b|01\rangle)|0\rangle \\
=\left|\left(\varphi^{2}\right)_{0}\right\rangle|0\rangle .
\end{gathered}
$$

If the measured first qubit state is 1 , then the state will be

$$
\begin{gathered}
|\xi\rangle_{1}=a|100\rangle+b|110\rangle=(a|10\rangle+b|11\rangle)|0\rangle \\
=\left|\left(\varphi^{2}\right)_{1}\right\rangle|0\rangle .
\end{gathered}
$$

Thus, after measuring the first qubit in the 3 -qubit state $|\xi\rangle$, in the first two qubits of the measurement we obtain one of the states of the doubled qubits $|\varphi\rangle^{2}$, namely

$$
\left|\left(\varphi^{2}\right)_{0}\right\rangle=a|00\rangle+b|01\rangle
$$

or

$$
\left|\left(\varphi^{2}\right)_{1}\right\rangle=a|10\rangle+b|11\rangle .
$$

The last qubit of both measurements is 0 . The full circuit of processing the given qubit $|\varphi\rangle$ and measuring the doubled qubits nested in the 3 -qubit state is shown in Fig. 5 (in Appendix). The parameter of measurement $M=0$ or 1 when the measured first qubit is 0 or 1 , respectively.
Thus, this circuit shows that the doubled qubits can be nested in the 3-qubit state, namely in the first two qubits of this state. The output of this scheme is a kind of shell containing doubled qubits, from where they can be measured.

\section{Algorithm of nesting and measuring the doubled qubits in the 3-qubit state:}

$$
\begin{array}{ll}
\text { 1. } & |\psi\rangle=X(|\varphi\rangle \oplus|0\rangle) . \\
\text { 2. } & |\phi\rangle=H|0\rangle . \\
\text { 3. } & |\chi\rangle=X[|\phi\rangle,|\psi\rangle]_{2} . \\
\text { 4. } & |\xi\rangle=P_{(1,5),(2,6)|\chi\rangle .} \\
\text { 5. } & \text { Measurement: }|\xi\rangle \rightarrow\left\{(1-M)\left|\left(\varphi^{2}\right)_{0}\right\rangle+\right. \\
& \left.M\left|\left(\varphi^{2}\right)_{1}\right\rangle,|0\rangle\right\} .
\end{array}
$$

6. The doubled qubits are described by the first two qubits of the measured 3-qubit,

$$
\begin{aligned}
|\varphi\rangle^{2} \approx|\xi\rangle_{M} & =(1-M)[\underbrace{a|00\rangle+b|01\rangle}] \\
+ & M[\underbrace{a|10\rangle+b|11\rangle}],
\end{aligned}
$$

when $M=0$ or 1 .

Each of these measured 2-qubit superpositions carries information of the original qubit $|\varphi\rangle$;

$$
\begin{gathered}
|\xi\rangle_{0}=a|00\rangle+b|01\rangle=|0\rangle|\varphi\rangle \text { and }|\xi\rangle_{1}= \\
a|10\rangle+b|11\rangle=|1\rangle|\varphi\rangle .
\end{gathered}
$$

Measuring any of these 2-qubits, we obtain the original qubit $|\varphi\rangle$.

\section{Conclusion}

The main challenges in quantum computing are not only in developing algorithms and quantum circuits. The accurate measurement of the calculated multiqubit state is also a difficult task to be solved. Unlike the calculation in the traditional computer, in quantum computing, many measurements are required, i.e., the circuit should be run repeatedly. The quantum circuits to get only the copy of the qubit, i.e., the doubled qubits, are unknown, namely, such circuits do not exist, according to what is said in the current literature. In this paper, we present a quantum circuit with CNOT operations, Hadamard gate, permutation and measurement, which shows the doubled qubits in two qubits of the calculated 3-qubit state. In other words, we have shown that there exist quantum schemes that allow us to measure doubled qubits. As stated in the introduction, in this work we presented our vision of transforming and copying qubits. 


\section{Appendix}

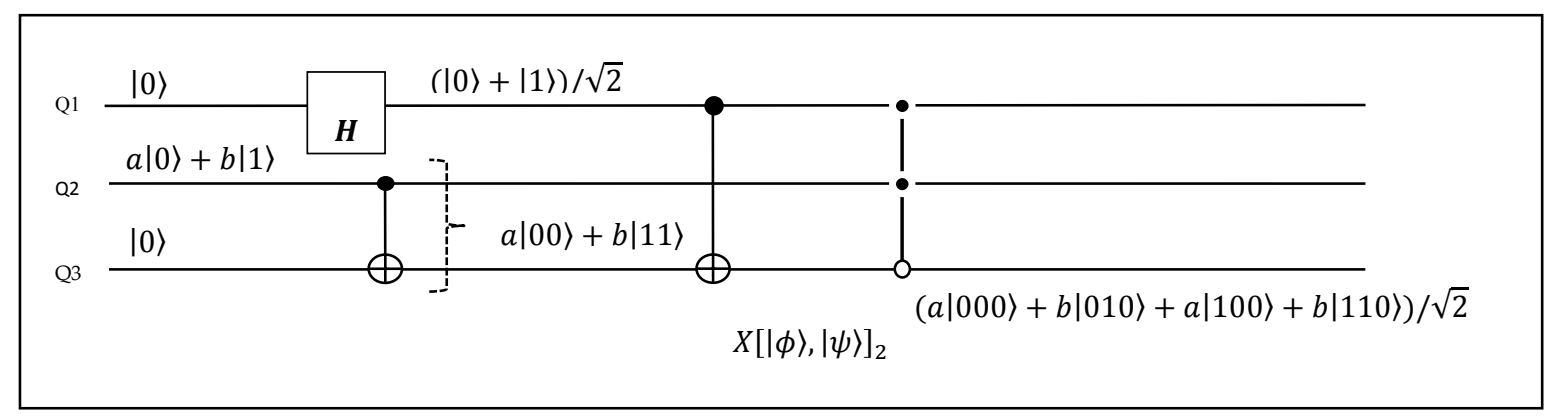

Figure 3 The 3-qubit circuit with two CNOT and 2-XOR operations.

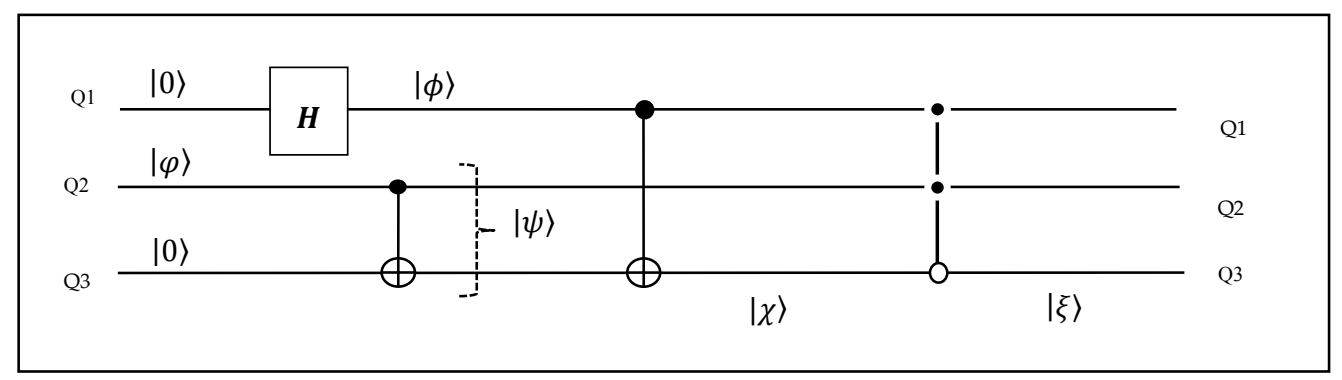

Figure 4 The 3-qubit circuit with two CNOT operations and 2-XOR operations.

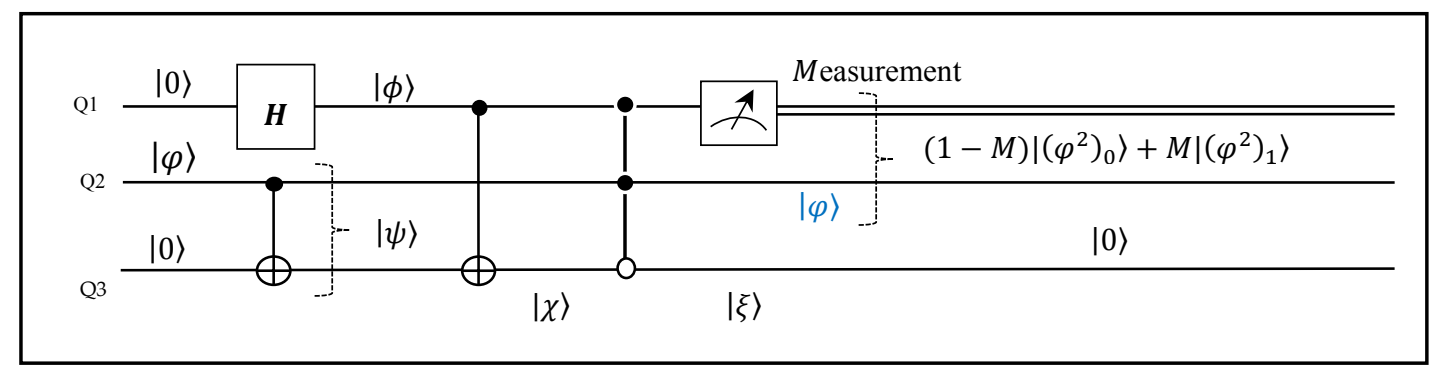

Figure 5 The 3-qubit circuit with measurement of the duplicated qubit. 
References:

[1] W.K. Wootters, W.H. Zurek, A single quantum cannot be cloned, Nature, vol. 299, 802-803 (1982).

[2] D. Dieks, Communication by EPR Devices, Phys. Lett., vol. 92A, 271-272 (November 1982)

[3] HR. Li, MX. Luo, H. Lai, Generalized quantum no-go theorems of pure states, Quantum Information Processing 17 (7), 168, p. 18 (2018).

[4] A.E. Rastegin, No-cloning theorem for a single POVM, Quantum Information and Computation, vol.10, no.11\&12, pp. 0971-0980 (2010).

[5] M. Nielsen, I. Chuang, Quantum Computation and Quantum Information, 2nd Ed., New York, Cambridge UP (2001).

[6] E. Strubell, An Introduction to Quantum Algorithms, Springer, vol. COS498 (2011).

[7] A. Peres, Quantum Theory: Concepts and Methods, Kluwer Academic Publishers Dordrecht, The Netherlands (1995).

[8] C.H. Bennett et al., Purification of noisy entanglement and faithful teleportation via noisy channels, Phys. Rev. Lett. 76, 722 (1996).

[9] XQ. Xiao, JM. Liu, Scheme for assisted cloning an unknown arbitrary three-qubit state, Quantum Information Processing 10, 567-574 (2011)

[10] V. BuzÏlek1, M. Hillery, P.L. Knight, Flocks of quantum clones: Multiple copying of qubits, Fortschr. Phys. 46, 4-5, 521-533 (1998)

[11] Xi-Jun Ren and H. Fan, Quantum circuits for asymmetric 1 to $n$ quantum cloning, Quantum Information and Computation, vol. 15, no. 11\&12, pp. 0914-0922 (2015).

[12] R. Jozsa, A stronger no-cloning theorem, (2002) arXiv:quant-ph/0204153v2

[13] V. Buzek, M. Hillery, Quantum copying: Beyond the no-cloning theorem, Phys. Rev. A 54, 1844 (1996).

[14] L. Duan, G. Guo, A probabilistic cloning machine for replicating two non-orthogonal states, Phys. Lett. A 243, 261 (1998).

[15] H. Fan, B. Liu, and K. Shi, Quantum cloning of identical mixed qubits, Quantum Information and Computation, vol. 7 , no. $5 \& 6$, pp. 551-558 (2007).
[16] Kar, G., Rahaman, R.: Local cloning of multipartite entangled states. Quantum Information Processing 11, 711-727 (2012).

[17] D. Leermakers and B. Skoric, Qubit-based unclonable encryption with key recycling, Quantum Information and Computation, vol. 21, no.11\&12, pp. 901-930 (2021).

[18] C. Bennett, G. Brassard, Quantum cryptography: Public key distribution and coin tossing, in Proc. of the IEEE Int. Conf. on Computers, Systems, and Signal Processing, Bangalore, 175-179 (1984).

[19] V. Scarani, S. Iblisdir, N. Gisin, Quantum cloning, (2005) https://arxiv.org/pdf/quantph/0511088.pdf

[20] I.B. Djordjevic, Quantum circuits and quantum information processing fundamentals, in Quantum Information Processing and Quantum Error Correction, 91-117 (2012).

Contribution of individual authors to the creation of a scientific article (ghostwriting policy)

Artyom Grigoryan: Conceptualization, Methodology, Circuits and Algorithm, Original draft preparation.

Sos Agaian: Problem formulation, Methodology, Original draft preparation and structuring.

Sources of Funding for Research Presented in a Scientific Article or Scientific Article Itself

'Not applicable'

\section{Conflicts of interest/Competing interests}

The author declares no conflicts of interest regarding the publication of this paper

\section{Creative Commons Attribution License 4.0 (Attribution 4.0 International, CC BY 4.0)}

This article is published under the terms of the Creative Commons Attribution License 4.0 https://creativecommons.org/licenses/by/4.0/deed.en US 